\title{
DENSIDAD Y DISTRIBUCIÓN DE UNGULADOS SILVESTRES EN LA RESERVA ECOLÓGICA EL EDÉN, QUINTANA ROO, MÉXICO
}

\author{
Rosa María González-MARín', Sonia Gallina1, Salvador MANduJAno1 \\ \& Manuel WeBER ${ }^{2}$ \\ ${ }^{1}$ Departamento de Biodiversidad y Ecología Animal, Instituto de Ecología A. C., Km. 2.5 \\ Carr. Ant. Coatepec No. 351, Xalapa 91070, Veracruz, MÉXICO. \\ gonzalez_rosy@hotmail.com \\ ${ }^{2}$ El Colegio de la Frontera Sur (ECOSUR). Unidad Campeche. \\ Calle 10 x 61 N0. 264. Colonia Centro. C. P. 24000. Campeche, CAMPECHE.
}

\section{RESUMEN}

Las selvas del noreste de la Península de Yucatán albergan poblaciones de ungulados silvestres como el venado cola blanca (Odocoileus virginianus Hays 1874), temazates (Mazama americana Erxleben 1777 y M. pandora Merriam 1901) y pecarí de collar (Pecari tajacu Merriam 1901) por lo que para su conservación y posible aprovechamiento es necesario evaluar el estado de las mismas. En el presente estudio se evaluaron aspectos ecológicos como la densidad y distribución en distintos tipos de vegetación de las poblaciones de estos ungulados silvestres en la Reserva Ecológica El Edén (REE), al norte de Quintana Roo. Se aplicó el método de conteo directo de animales en transectos de franja en senderos existentes. Mediante Sistemas de Información Geográfica se clasificó el hábitat en cinco tipos de vegetación: acahual, selva, tintales, sabana de palmas y vegetación acuática. Se obtuvieron 38 observaciones de venado cola blanca, 14 de temazates y 6 grupos de pecaríes en 293.4 $\mathrm{km}$ de recorridos repartidos en 6 meses de muestreos diurnos entre septiembre de 2004 y abril de 2005. La densidad absoluta del venado cola blanca fue de $5.5 \pm 4.1 \mathrm{ind} / \mathrm{km}^{2}$, para el pecarí de collar fue $1.9 \pm 0.8 \mathrm{ind} / \mathrm{km}^{2}$ o $0.2 \pm 0.1$ manadas $/ \mathrm{km}^{2}$ y para los temazates fue de $1.7 \pm 1.8 \mathrm{ind} / \mathrm{km}^{2}$. El acahual fue el tipo de vegetación donde se distribuyen más frecuentemente el venado cola blanca y el pecarí de collar. Este trabajo registró por primera vez a Mazama americana para esta región de Quintana Roo. Los resultados sugieren que la REE tiene un papel importante en la conservación de los ungulados, ya que a pesar de la perturbación continua por incendios y huracanes, en la REE se tiene una densidad relativamente alta, principalmente del venado cola blanca en comparación con otros sitios tropicales.

Palabras clave: Ungulados, densidad, distribución, vegetación, la REE, Quintana Roo.

\begin{abstract}
The forests of the northeast Yucatan Peninsula supports wild ungulate populations such as the white-tailed deer (Odocoileus virginianus (Hays 1874), brocket deer (Mazama americana Erxleben 1777 and M. pandora Merriam 1901) and collared pecari (Pecari tajacu Merriam 1901). For its conservation and use, it is necessary to know the current status of wild ungulates populations. Basic
\end{abstract}


ecological aspects such as density and distribution of the ungulate populations in different vegetation types were evaluated. The study area was El Edén Ecological Reserve (EER), located in the northern Yucatan Peninsula in Quintana Roo State, Mexico. The method used to assess ungulate density and distribution was the strip transect counts. The transects were set on the existing roads and footpaths. Using GIS, the habitat and five vegetation types were classified: secondary vegetation (acahual), tropical evergreen forest, tintales, savanna of palms and aquatic vegetation. Thirty eight observations of white-tailed deer were obtained, 14 of brocket deer and 6 groups of pecaries in $293.4 \mathrm{~km}$ of routes. The observation period was between September 2004 and April 2005 (six months of sampling). The absolute density of the white-tailed deer was $5,5 \pm 4,1 \mathrm{ind} / \mathrm{km}^{2}$, for the pecari was $1,9 \pm 0,8 \mathrm{ind} / \mathrm{km}^{2}$ or $0,2 \pm 0,1 \mathrm{herd} / \mathrm{km}^{2}$ and for brocket deer was $1,7 \pm 1,8 \mathrm{ind} / \mathrm{km}^{2}$. Acahual was the vegetation type where white-tailed deer and collared pecari were found. This is the first time that M. americana was registered in this region of the State. These results suggest the important role that have the EER to conserve ungulates, nevertheless the continued risk of hurricanes and fires. The white-tailed deer population is high compared with other tropical zones.

Key words: Ungulates, density, distribution, vegetation, the EER, Quintana Roo.

\section{INTRODUCCIÓN}

Las selvas de la Península de Yucatán son el resultado de la manipulación humana y fenómenos naturales. Hay eventos naturales tales como huracanes e incendios que afectan grandes áreas de selva tropical. También actividades humanas como la agricultura, ganadería y urbanización que modifican las selvas (Gómez-Pompa et al. 2003). Sin embargo, estas selvas pueden albergar una gran diversidad de fauna silvestre, y para su conservación y posible aprovechamiento es necesario saber en que estado se encuentran las poblaciones que viven en ellas. Como primer paso, son necesarios estudios sobre ecología de poblaciones donde puedan conocerse su demografía, su relación con otras especies y su distribución en el hábitat. Para esto, se seleccionaron a los ungulados como grupo de estudio porque tienen un alto significado cultural en la Península de Yucatán, ya que han sido objeto de cacería de subsistencia desde épocas prehispánicas, sirviendo como una importante fuente de proteína en la alimentación de grupos indígenas y poblaciones rurales (Mandujano \& Rico-Gray 1991, Naranjo et al. 2004). Así mismo, han sido muy importantes en la cosmogonía maya y en su mitología (De Landa 1938). También, los ungulados son las principales presas del jaguar (Panthera onca) y del puma (Puma concolor), por lo que su presencia en un determinado sitio es indicador de la existencia de grandes felinos (Aranda 2002, Olivera 2002). Estos animales también pueden ejercer un efecto en la estructura de la vegetación de las selvas con el ramoneo que realizan y su participación en la dispersión de semillas (Dirzo \& Miranda 1991, Fragoso 1997).

El estudio de los ungulados se ha incrementado notablemente en las regiones tropicales en los últimos años (E.g., Naranjo 2002, Mandujano et al. 2004), sin embargo, la información sobre ecología de las poblaciones de ungulados en la 
Península de Yucatán es escasa y hasta hace unos pocos años comenzaron a realizarse investigaciones en ese tema (Reyna- Hurtado 2002, Ávila 2003, Weber 2005). Hasta ahora, en la REE no existían investigaciones sobre las poblaciones de ungulados, por lo que este trabajo fue relevante para dar a conocer las especies de ungulados en la Reserva y contribuir al conocimiento ecológico de sus poblaciones. Así mismo, aportó información sobre la importancia de las reservas ecológicas pequeñas como estrategia de conservación de la vida silvestre (Gómez-Pompa \& Dirzo 1995). La información generada también servirá de base para estudios ecológicos más especializados y enfocados al manejo y conservación de ungulados en el futuro. El objetivo del presente estudio fue estimar la densidad y distribución por tipo de vegetación de las poblaciones de ungulados en la Reserva Ecológica El Edén, Quintana Roo, México.

\section{ÁREA DE ESTUDIO}

La REE fue establecida en 1990, y es una de las primeras reservas privadas dedicadas a la investigación para la conservación biológica en México. Antiguamente la zona se utilizaba para la explotación forestal y cultivo del chicle. Más adelante parte de esta misma zona fue utilizada para la ganadería. Después de estas actividades, el área estuvo varios años sin tener uso alguno, y comenzó un proceso de regeneración. Fue hasta entonces, que un grupo de conservacionistas interesados en el trópico Maya crearon en esta zona, un área protegida experimental (Gómez-Pompa et al. 2003). La REE es un sitio que permite generar modelos de procesos ecológicos, tales como los que se han realizado en la Estación biológica "La Selva" de Costa Rica, o en la Isla de Barro Colorado en Panamá (Allen \& Rincón 2003).

La REE, está situada al norte del Estado de Quintana Roo, en el Municipio de Lázaro Cárdenas, en el Noreste de la Península de Yucatán, a los $21^{\circ} 36^{\prime}$ y $20^{\circ} 34^{\prime}$ de latitud $\mathrm{N}$ y a los $87^{\circ} 06^{\prime}$ y $87^{\circ} 45^{\prime} \mathrm{W}$ de longitud Oeste, y a una altitud de 5 a $10 \mathrm{msnm}$ (Fig. 1). La entrada está a la altura del kilómetro 290 de la carretera Mérida a Cancún, al norte de Leona Vicario, en donde se inicia hacia el norte un camino de tercería de unos $10 \mathrm{~km}$. Cuenta con una extensión de más de 1,492 ha. La REE pertenece a la región biológica conocida como Yalahau, que significa en Maya "donde brota o nace el agua" (Gómez-Pompa 2000, Gómez-Pompa et al. 2003).

La vegetación del Edén puede ser dividida en 5 tipos (Schultz 2003): (a) la selva mediana (vegetación de estructura mediana, semidecidua); esta selva ha sido fuertemente perturbada por los frecuentes huracanes provenientes del Caribe que cruzan el norte de la Península de Yucatán, por las quemas que han ocurrido desde épocas muy antiguas y por las explotaciones forestales que se han hecho desde el siglo antepasado. Esta formada por árboles que alcanzan una altura de $10 \mathrm{a} 15 \mathrm{~m}$. Los 
González Marín et al.: Densidad y distribución de ungulados silvestres

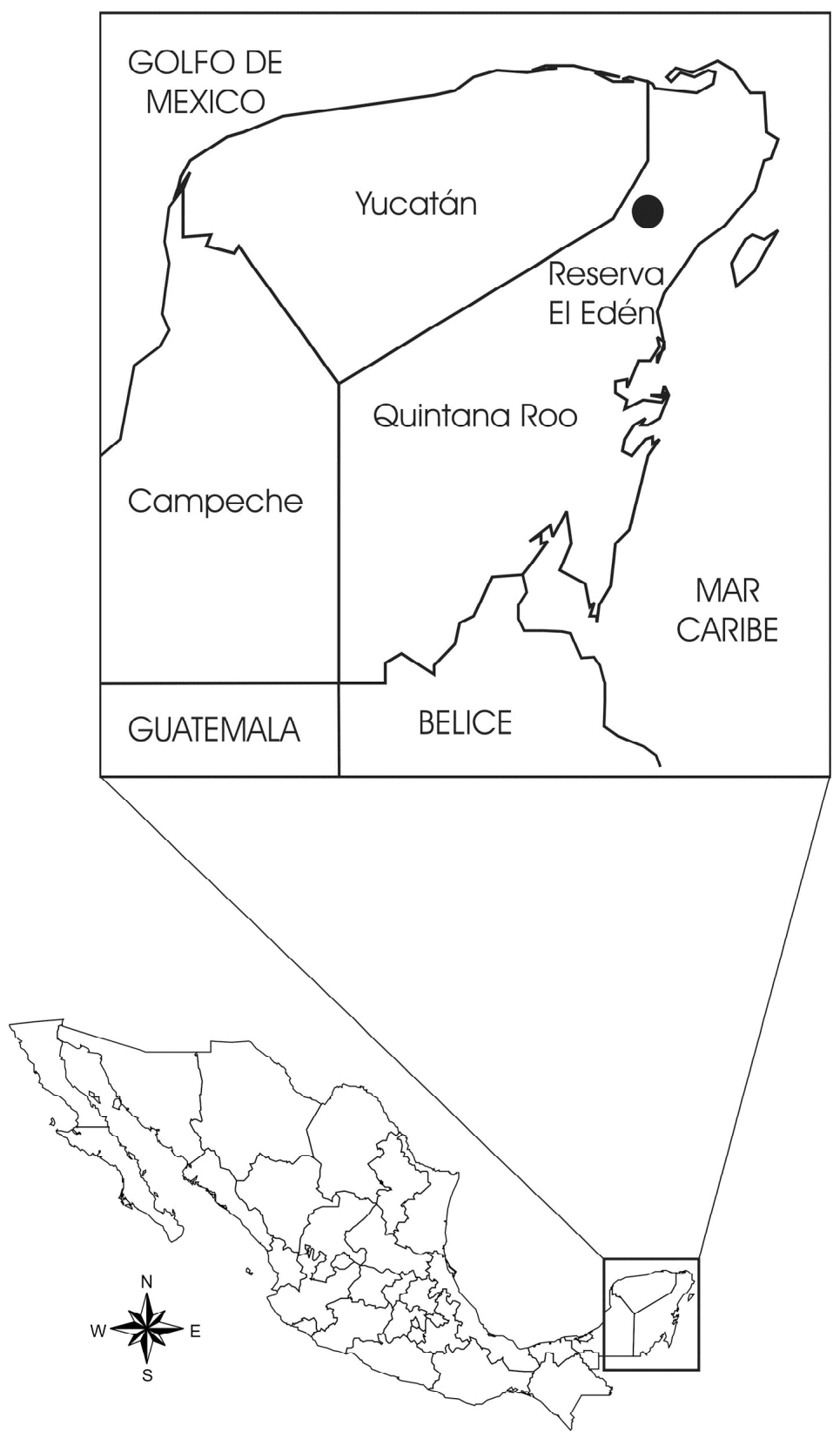

Figura 1

Situación geográfica de la REE, en el Estado de Quintana Roo, al Este de Yucatán y al Noreste de Campeche, en la Península de Yucatán. 
árboles con mayor importancia en la selva son Manilkara zapota, Lysiloma latisiliqua, Metopium brownei y la palma Thrinax radiata. (b) los acahuales de diferentes edades, (refiriéndose a vegetación secundaria a consecuencia de disturbios); es la vegetación derivada de la selva mediana con árboles de 3 a 6 metros de altura en los acahuales jóvenes y de 7 a 10 metros en los acahuales viejos. Los árboles de mayor importancia en la vegetación secundaria son Metopium brownei, Bursera simaruba, y Swartzia cubensis. En el acahual la densidad en ramaje es mayor que en selvas. (c) bosque inundable (tintales y manchones de anonáceas que crecen en sitios inundables); el tintal es bajo en estructura (menos de $10 \mathrm{~m}$ ). Está estacionalmente inundado entre 4 y 6 meses en el año. Es dominado por Haematoxylon campechianum. Así mismo, Erythroxylon confusum y Byrsonima bucidaefolia son también muy comunes. En la parte norte de la reserva se encuentran dos pequeños bosques de anonáceas. Estas comunidades se encuentran como grupos de una sola especie de árbol (Annona glabra) con un grupo de helechos (Achrostichum danaefolium). Estos están rodeados por un denso grupo de Typha dominguensis, Phragmites australis y Sagitaria lancifolia. (d) sabanas (que pueden ser áreas abiertas con palmas); se encuentran intermedias entre el tintal y los pantanos de Cladium y están dominadas por Acoeloraphe wrightii con individuos dispersos del árbol leñoso Crescentia cujete. También Acoeloraphe wrightii es una pequeña palma que forma grupos, los cuales pueden ser de 2 a 15 individuos. Hay epifitas presentes en las palmas, mayormente orquídeas Carasetum integgerinum y Mymecophila aff. christinii, y (e) vegetación acuática (que crece en zonas pantanosas o cerca de los cenotes); la mayoría de los pantanos en la reserva están dominados por Cladium jamaicensis, un pasto cortador que puede medir de 2 a $3 \mathrm{~m}$ de alto. Pantanos dominados por Typha dominguensis son encontrados en depresiones con suelos más profundos que son anualmente húmedos excepto en los años con mucha sequía. El tercer tipo de pantano está dominado por Eleocharis cellulosa y Rhynchospora holoschoenoides. Esas áreas tienden a ser pobres en especies, con solo algunos individuos ocasionales de Nymphoides indica y Centella asiática.

\section{MÉTODO}

\section{Registro de datos}

El registro de información se realizó en seis muestreos de campo (15 días por mes), tres meses en lluvias (julio, agosto y noviembre de 2004) y tres meses en secas (febrero, marzo y abril de 2005). El área cubierta con los muestreos respecto a la extensión de la reserva fue del $40 \%$. Los muestreos se efectuaron por el método de transecto de franja (Robinette et al. 1974). Los transectos se realizaron sobre los cuatro senderos existentes en la reserva ya que era lo más seguro y factible para 
obtener registros. Aunque los transectos deberían ubicarse de manera aleatoria, fue poco práctico, ya que por lo denso de la vegetación se tendrían que haber chapeado varios kilómetros de vegetación natural y esto no se permite en la REE por ser un sitio protegido y en regeneración. Suponiendo que se hubiera hecho, aun así habría otros inconvenientes, como la visibilidad baja por la densa vegetación del sotobosque alrededor de los transectos que provocarían datos sesgados. Así mismo, el ruido al pisar la hojarasca seca, tendría como consecuencia que los ungulados que estuvieran en el interior del transecto corrieran antes de ser detectados. Este último punto violaría unos de los supuestos del método de transecto de franja que menciona que todos los animales que estén dentro la franja deben ser detectados (Robinette et al. 1974). Otro inconveniente para los muestreos, es que gran parte de la reserva se encuentra inundada permanentemente con piedras resbaladizas, esto es peligroso para la persona que va observando animales. Por lo tanto, aunque reconocemos que el empleo de senderos tiene limitaciones, en el presente estudio se decidió emplearlos para ubicar los transectos de muestreo.

Los senderos fueron divididos en varios transectos clasificados por tipos de vegetación y se incluyeron cinco formaciones vegetales: selva mediana, acahuales de diferentes edades, tintales, sabanas con palmas, y vegetación acuática. Los recorridos se hicieron de 0700 a 1200 y de 1500 a 1900 h. Los transectos fueron caminados por una persona y en ocasiones con un acompañante. Cuando un individuo de venado cola blanca, temazate o pecarí era detectado se registraban los siguientes datos: número de sendero, tipo de vegetación dominante, número de recorrido, fecha, hora, especie observada, sexo, edad, número de animales observados (en el caso de venados juntos por estar en etapa reproductiva o con crías), y posición sobre el transecto con un Sistema de Posicionamiento Global (GPS). En el caso de los temazates (Mazama americana y M. pandora), se decidió registrarlos a nivel de género (Mazama spp.) ya que sólo se pudieron identificar algunos individuos hasta especie. Cuando la observación no era clara entre venados temazates y venado cola blanca se clasificaba solamente como venado, sin embargo estos datos no fueron incluidos en los análisis.

La clase de edad en los individuos se determinó de la siguiente manera: adultos, juveniles y crías. Esto se realizó por observaciones del tamaño del animal, por ejemplo, en machos adultos corpulencia gruesa y machos juveniles corpulencia delgada, también mediante el tamaño de las astas y la coloración, en caso de hembras, color café y corpulencia delgada y las crías con pintas blancas o también si se encontraba con la madre. Para clasificar el sexo de los venados se observó coloración entre machos y hembras en caso del cola blanca (machos grises y hembras café rojizo), así como presencia o ausencia de astas en cola blanca y temazates, en el caso de las crías no se determinó. Esta determinación solo se pudo hacer con los venados, sin embargo sólo se determinó en algunos individuos. Para los pecaríes no 
fue posible diferenciar pues es difícil distinguir el sexo, sin embargo sí se determinaron en adultos, juveniles y crías. En el caso de los animales observados en repetidas ocasiones (a juzgar por el sexo, edad y ubicación en los senderos) estos datos fueron eliminados de los análisis. En el caso de los rastros que fueron encontrados, se posicionaron con GPS. Sin embargo, como en campo no se contaba con un experto en rastros que permitiera la sistematización del método, así como la identificación precisa de la especie a la cual correspondían, éstos solo fueron utilizados como apoyo en la evaluación de la presencia de los ungulados en los diferentes tipos de vegetación pero no fueron incluidos en los análisis.

\section{Clasificación de la vegetación}

El tipo de vegetación fue clasificado por medio de Sistemas de Información Geográfica (SIG). Esto se realizó mediante un mapa de la vegetación que se digitizó con el programa ArcView 3.2, basado en los manchones de vegetación de dos ortofotos originadas de una fotografía aérea tomada en 2001 a una escala 1:75000 y un trabajo de tesis sobre la vegetación de la REE (Schultz 2003), el cual incluye una descripción y mapa esquemático de los diferentes tipos de vegetación de la REE. Cinco tipos de vegetación fueron clasificados sobre el mapa y se determinó su superficie (lo que representa su disponibilidad). Así mismo, los diferentes tipos de vegetación correspondientes en cada transecto fueron medidos utilizando coordenadas tomadas con GPS como referencia. El GPS que se utilizó para los muestreos fue un modelo eTrex Venture de Garmin, con 15 m de precisión y Datum UTM 83.

\section{Análisis de datos}

Densidad. - Para obtener la densidad aplicando el modelo de franja, se dividió el número de individuos observados en cada transecto entre el largo del transecto por tipo de vegetación, multiplicado por el ancho establecido $\mathrm{w}=10 \mathrm{~m}$. La densidad y su desviación estándar, fueron estimadas para cada mes de muestreo. La densidad total se obtuvo promediando los valores de cada mes. Se tomó la medida del ancho con menor visibilidad estimada más frecuente en el sotobosque para todos los transectos, ya que los diferentes tipos de vegetación de la REE se mezclan fácilmente entre sí, y muchas veces en un mismo tipo de vegetación uno podía tener mayor visibilidad y otras veces menor, por lo que se decidió estandarizar con el tipo de vegetación de menor visibilidad (acahual) para garantizar la observación de todos los animales dentro de la franja. Así mismo, para el análisis se tomó en cuenta el número de recorridos realizados en el muestreo. Se realizó una prueba de normalidad de los datos. Al ver que los datos tenían una distribución tipo Poisson se utilizó estadística no paramétrica. Para saber si existían diferencias de densidad entre especies y entre tipos de vegetación, se utilizó la prueba Kruskal Wallis (Zar 1996). Para aplicar las pruebas y análisis, se usó el paquete estadístico JMP IN Versión 4 (SAS Institute Inc. 2000). 


\section{Distribución por tipos de vegetación}

Utilizando el programa ArcView 3.2, fueron posicionados los puntos de observaciones de ungulados sobre el mapa de vegetación digitalizado y con la función Geoprocesing se realizó una intersección de los puntos posicionados de ungulados para identificar en que tipo de vegetación se encontraba cada uno de los individuos.

La distribución por tipos de vegetación fue definida como el número total de individuos de cada especie obtenido en cada tipo de vegetación durante todo el muestreo. Para estimar en que tipo de vegetación existía una mayor distribución de ungulados se empleó una prueba de $X^{2}$ (Zar 1996) y los intervalos de Bonferroni al $95 \%$ de confianza para estimar las preferencias por determinado tipo de vegetación (Byers et al. 1984).

\section{RESULTADOS}

Se registraron en total 38 observaciones de venado cola blanca (Odocoileus virginianus yucatanensis), 14 observaciones de venado temazate (Mazama spp.) y seis grupos de pecaríes (Pecari tajacu yucatanensis) en $293.4 \mathrm{~km}$ de recorrido repartidos en seis meses de muestreo. El número de individuos por grupo de pecaríes fluctuó de 2 a 15, con una media aritmética y desviación estándar de $7.8 \pm 4.7$ individuos por grupo.

De los venados cola blanca observados, se identificaron 14 hembras, 11 machos y ocho crías, el resto no fue determinado. Con respecto a los temazates fueron identificados y fotografiados dos individuos de $M$. pandora. Así mismo, en este estudio se identificaron visualmente tres individuos de $M$. americana, siendo éstos los primeros registros en el área norte de Quintana Roo. De los temazates se identificaron siete hembras, cinco machos y una cría.

El tipo de vegetación donde se observó un mayor número de individuos fue el acahual, en segundo lugar correspondió a los tintales y la selva, y el menor número de individuos se observó en las sabanas.

\section{Densidad}

Con respecto a la densidad absoluta, la prueba estadística demostró diferencias significativas entre especies $\left(\mathrm{H}=7.4805 \chi^{2} 0.05(2)=5.991, P<0.05\right)$. El venado cola blanca es el ungulado con mayor densidad $\left(5.5 \pm 4.1 \mathrm{ind} / \mathrm{km}^{2}\right)$, en segundo lugar está el pecarí de collar $\left(1.9 \pm 0.8 \mathrm{ind} / \mathrm{km}^{2} ; 0.25 \pm 0.10\right.$ grupos $\left./ \mathrm{km}^{2}\right)$ y el temazate $(1.7 \pm$ $1.8 \mathrm{ind} / \mathrm{km}^{2}$ ) es el de menor densidad.

Analizando a las poblaciones de ungulados en conjunto, se encontraron diferencias de densidad entre los tipos de vegetación $\left(\mathrm{H}=26.6338 \chi^{2}{ }_{0.05(4)}=9.4877\right.$, 
$P<0.05)$. El acahual es el tipo de vegetación con mayor densidad de ungulados, le siguen los tintales y la selva y las menores densidades se presentaron en las sabanas (Fig. 2). Analizando por especie, no se encontraron diferencias en la densidad de venado cola blanca con respecto a los tipos de vegetación del Edén $(\mathrm{H}=6.6399$ $\left.\chi^{2} 0.05(4)=9.4877, P>0.05\right), \mathrm{y}$ tampoco para los temazates $\left(\mathrm{H}=3.8810 \chi^{2} 0.05(2)=\right.$ 5.991, $P>0.05)$. Se encontraron diferencias en la densidad de pecarí de collar entre el acahual y la selva del Edén $\left(U_{(0.05,2)}=2.162, P<0.05\right)$. Los resultados mostraron un mayor número de individuos por $\mathrm{km}^{2}$ en el acahual que en la selva (Fig. 3).
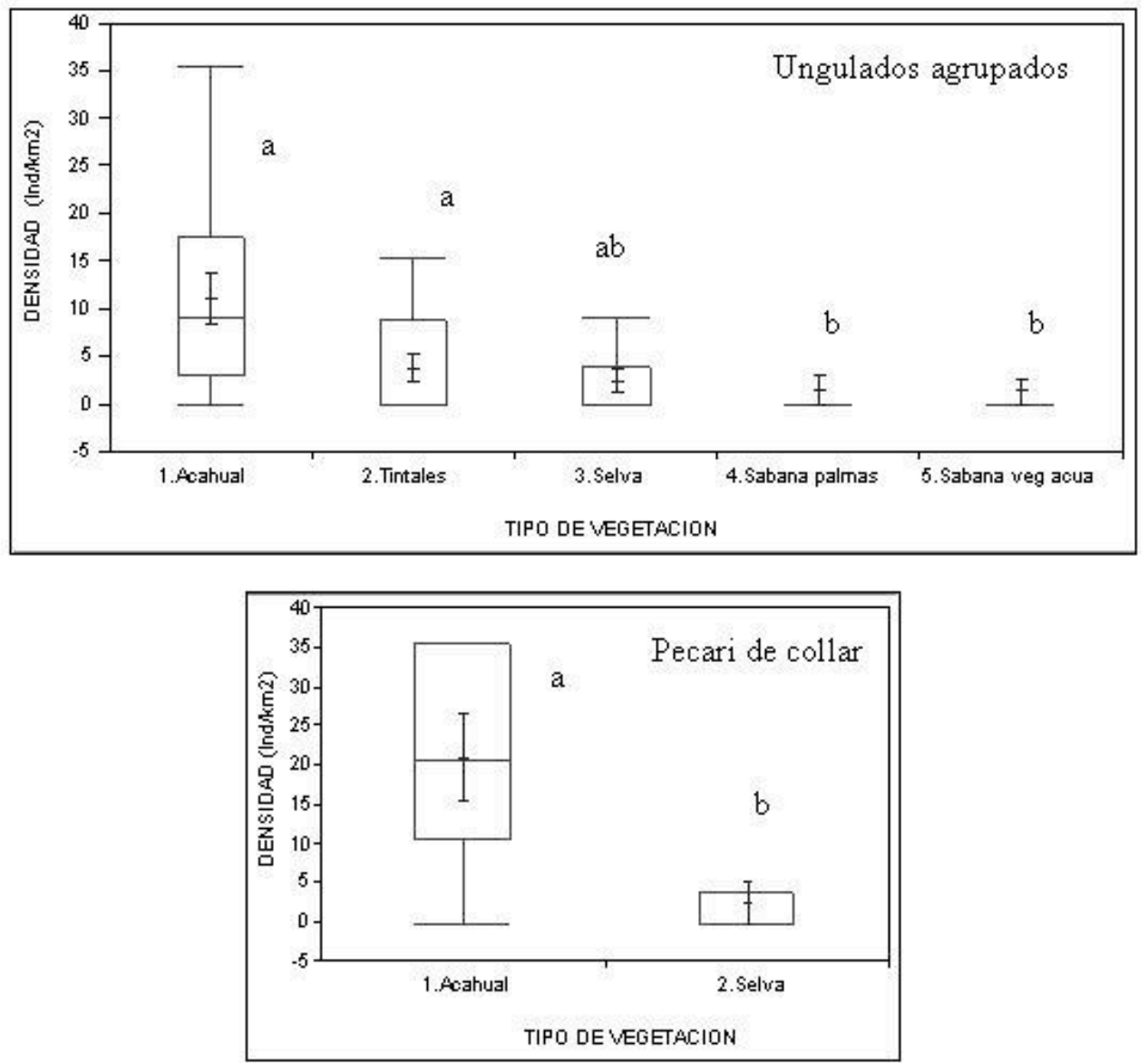

Figura 2

Diferencias significativas de densidad para los ungulados en conjunto y para el pecari de collar, con respecto a los tipos de vegetación de la REE. Las líneas simbolizan el promedio con su error estándar y las barras reflejan la dispersión de los datos. Las letras diferentes indican diferencias estadísticamente significativas. 


\section{Distribución de los ungulados en base a los tipos de vegetación}

Se encontraron diferencias significativas en la distribución del venado cola blanca con respecto a los tipos de vegetación $\left(\chi^{2}=36.93\right.$, g.l. $=4, P<0.05$; Cuadro 1$) \mathrm{y}$ del pecarí de collar $\left(\chi^{2}=46.06\right.$, g.l. $=4, P<0.05$; Cuadro 1$)$, donde el acahual fue el tipo de vegetación más frecuentado, mientras que las sabanas fueron menos frecuentadas por el venado cola blanca (Fig. 3). Los pecaríes no estuvieron presentes en las sabanas ni en los tintales. No se encontró diferencia en cuanto a la distribución de los temazates $\left(\chi^{2}=8.51\right.$, g.1. $=4, P>0.05$; Fig. 3$)$.

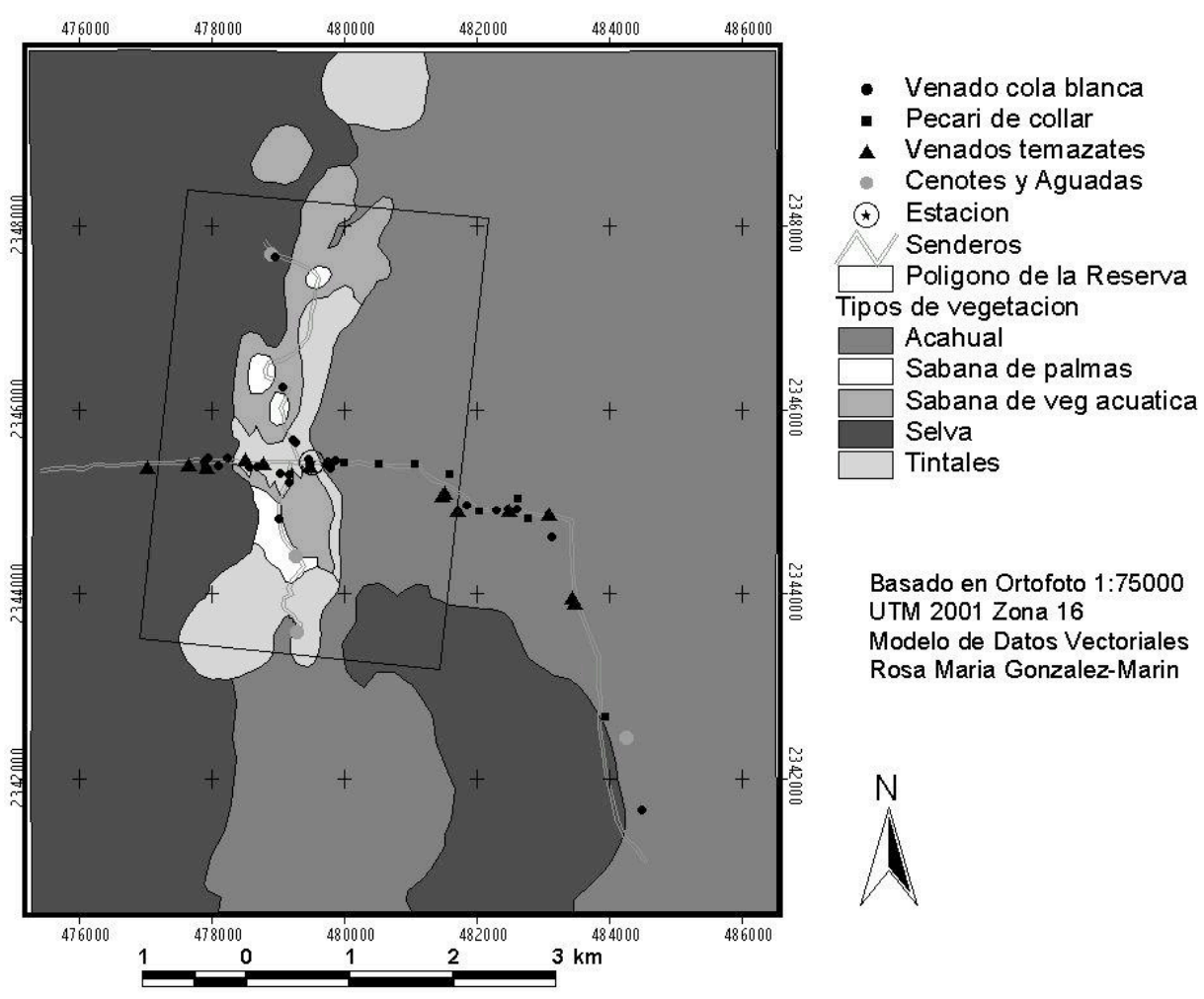

Figura 3

Distribución del venado cola blanca, venados temazates y pecarí de collar en la REE, Quintana Roo, México. Las localizaciones representan los sitios de observaciones georeferenciadas. 


\section{Cuadro 1}

Diferencias en la distribución del venado cola blanca y el pecari de collar con respecto a los tipos de vegetación $\left(\chi^{2}=36.93\right.$, g.l. $=4, \mathrm{P}<0.05 ; \chi^{2}=46.06$, g.l. $\left.=4, \mathrm{P}<0.05\right)$. $\mathrm{Pe}=$ proporción esperada de individuos; $\mathrm{Pi}=$ proporción observada de individuos.

\begin{tabular}{|c|c|c|c|c|c|c|}
\hline \multirow{2}{*}{$\begin{array}{c}\text { Tipo de } \\
\text { vegetación }\end{array}$} & \multicolumn{3}{|c|}{ Venado cola blanca } & \multicolumn{3}{|c|}{ Pecarí de collar } \\
\hline & $\mathrm{Pe}$ & $\mathrm{Pi}$ & $\begin{array}{l}\text { Intervalos } \\
\text { Bonferroni }\end{array}$ & $\mathrm{Pe}$ & $\mathrm{Pi}$ & $\begin{array}{l}\text { Intervalos } \\
\text { Bonferroni }\end{array}$ \\
\hline Acahual & 0.372 & 0.526 & $\mathrm{Pi}>\mathrm{Pe}$ & 0.372 & 0.772 & $\mathrm{Pi}>\mathrm{Pe}$ \\
\hline Sabana de palmas & 0.108 & 0.053 & $\mathrm{Pi}<\mathrm{Pe}$ & 0.108 & --- & $\mathrm{Pi}<\mathrm{Pe}$ \\
\hline $\begin{array}{c}\text { Sabana de vegetación } \\
\text { acuática }\end{array}$ & 0.074 & 0.026 & $\mathrm{Pi}<\mathrm{Pe}$ & 0.074 & --- & $\mathrm{Pi}<\mathrm{Pe}$ \\
\hline Selva & 0.264 & 0.158 & $\mathrm{Pi}=\mathrm{Pe}$ & 0.264 & 0.228 & $\mathrm{Pi}=\mathrm{Pe}$ \\
\hline Tintales & 0.182 & 0.237 & $\mathrm{Pi}=\mathrm{Pe}$ & 0.182 & --- & $\mathrm{Pi}<\mathrm{Pe}$ \\
\hline
\end{tabular}

Pi> Pe: Mayor distribución de individuos de lo esperado. $\mathrm{Pi}<\mathrm{Pe}$ : Menor distribución de individuos de lo esperado. $\mathrm{Pi}=\mathrm{Pe}$ : Distribución de individuos según lo esperado.

\section{DISCUSIÓN}

\section{Densidad}

Los resultados de este estudio sugieren que el venado cola blanca es la especie de ungulado más abundante en la REE, en comparación con los temazates y el pecarí de collar. Es posible que al ser la REE un sitio perturbado compuesto mayormente por vegetación secundaria, podría estar favoreciendo la presencia de especies de hábitos generalistas, como el venado cola blanca y el pecarí de collar (Reyna-Hurtado 2002, Bello 2004, Weber 2005). Por el contrario, como sugieren Emmons y Feer (1990) y Weber (2005), los venados temazates podrían ser menos beneficiados en este lugar ya que generalmente habitan selvas con menor grado de perturbación.

La densidad del venado cola blanca estimada en la REE fue relativamente diferente en relación a otros estudios de México. Por ejemplo, en Chamela, Jalisco se reportan 10-14 ind $/ \mathrm{km}^{2}$ (Mandujano \& Gallina 1995); Naranjo (2002) obtuvo $0.002 \mathrm{ind} / \mathrm{km}^{2}$ en la Selva Lacandona, Chiapas; Ávila (2003) reporta 3-12 ind $/ \mathrm{km}^{2} \mathrm{al}$ sur de Quintana Roo; en Calakmul, Campeche Weber (2005) menciona 0.02 ind $/ \mathrm{km}^{2}$ y se han reportado también 0.7 huellas/km (Reyna-Hurtado \& Tanner 2007). Por lo tanto, la densidad de cola blanca en la REE es relativamente similar a lo que reporta 
Ávila al sur de Quintana Roo. Así mismo, la densidad de venado cola blanca en la REE es comparablemente mayor con estudios realizados en Centro y Sudamérica, como son por ejemplo, los de Wright et al. (2000), en Panamá reportando 0.93 ind $/ \mathrm{km}^{2}$ y Pacheco-Figueroa (2003) en Costa Rica reportando $<1 \mathrm{ind} / \mathrm{km}^{2}$.

La densidad de temazates en la REE difiere si se compara con otros estudios en México. Por ejemplo, Naranjo (2002) reporta 0.23 ind $/ \mathrm{km}^{2}$; Ávila (2003) reporta 2$8 \mathrm{ind} / \mathrm{km}^{2}$; Weber (2005) obtiene $0.09 \mathrm{ind} / \mathrm{km}^{2}$, y se reportan también 6.6 huellas $/ \mathrm{km}$ (Reyna-Hurtado \& Tanner 2007); teniendo mayor similitud con los estudios realizados en Centro y Sudamérica, lo que probablemente se deba a la similitud en la forma en que se obtuvieron los registros, ya que tanto en los trabajos de Barro Colorado, Panamá con 0.93 ind $/ \mathrm{km}^{2}$ (Wright et al. 2000), en la costa de Surinam con $1.0 \mathrm{ind} / \mathrm{km}^{2}$ (Brannan \& Marchington 1987) y Yavari-Miri, Perú con $1.15 \mathrm{ind} / \mathrm{km}^{2}$ (Hurtado-González \& Bodmer 1994) eran reservas y en algunas se utilizaron senderos para su registro a diferencia de otros estudios en México en donde los senderos se han evitado (Reyna-Hurtado 2002, Weber 2005).

Con respecto a la densidad del pecarí de collar estimada en la REE, es similar a la obtenida por Ávila (2003) y Merediz (1995) en Quintana Roo con 1-15 ind $/ \mathrm{km}^{2}$ y $0.17 \mathrm{ind} / \mathrm{ha}$ consecutivamente, y Naranjo (2002) en la Lacandona con $1.76 \mathrm{ind} / \mathrm{km}^{2}$. Mayor que la obtenida por Pacheco (2004) en Yucatán con $0.15 \mathrm{ind} / \mathrm{km}^{2}$ y en Calakmul con 0.9 huellas/km (Reyna-Hurtado \& Tanner 2007); y menor a la obtenida por Mandujano (1999) con $4.9 \mathrm{ind} / \mathrm{km}^{2}$ en Chamela, Jalisco.

En la REE el número de individuos por grupo o manada varía entre dos y 15 , siendo los grupos de cuatro a 10 pecaríes los más comunes. Estos grupos son similares a lo que Merediz (1995) y Ávila (2003) encontraron al sur de Quintana Roo. En particular, es más frecuente que formen grupos relativamente grandes (de cinco a 15 individuos) en el Acahual, mientras que en la selva los grupos son más pequeños (de dos a cuatro pecaríes; González-Marín 2006). Habría que realizar estudios más específicos de la especie para sugerir que estos pequeños grupos puedan ser subdivisiones de un grupo mayor, como se ha sugerido en estudios realizados en Chamela (Mandujano 1999). Así mismo, en el tiempo en que fue realizado el muestreo, los grupos estaban constituidos por animales adultos y jóvenes, observándose crías pequeñas en julio; a diferencia de Chamela, que las crías se observan mayormente entre octubre y noviembre (Mandujano 1999).

Con respecto a la densidad por tipos de vegetación, para los ungulados en conjunto fue significativamente mayor en la vegetación secundaria. Posiblemente porque el acahual es lo que más abunda en la REE y la mayoría de los individuos registrados fueron especies que les gusta este tipo de vegetación como lo son el venado cola blanca y el pecarí de collar. Sin embargo, la densidad por tipos de vegetación específicamente para el venado cola blanca y los temazates no fueron significativamente diferentes y esto probablemente sucedió porque el número de 
individuos observados para cada especie fue pequeño y al hacer el análisis con cada una de ellas no permitió detectar diferencias significativas. Hay que tomar en cuenta, que el tamaño de muestra pequeño se debió también a la no inclusión de algunos registros de individuos de venados que no pudieron identificarse a nivel especifico, lo que pudo haber influido en que los resultados de las pruebas estadísticas no fueran significativas. Sin embargo, haber incluido datos de individuos de este tipo, hubiera sesgado los resultados de manera importante, por lo que se prefirió disminuir el tamaño de muestra pero tener la certeza de trabajar con datos seguros. En otros estudios como el de Ávila (2003) muestra que la densidad por tipo de vegetación en el caso del venado cola blanca es mayor en la vegetación secundaria, por lo que quizás si se tuviera un número mayor de individuos para analizar se podría haber encontrado algo similar. Por lo tanto, habría que realizar mayor número de muestreos abarcando una mayor superficie, por más tiempo, para obtener más registros de cada una de las especies de ungulados. En relación al pecarí de collar, la densidad por tipo de vegetación fue mayor significativamente en el acahual y esto corresponde con lo que Merediz (1995) y Ávila (2003) encontraron también en Quintana Roo, donde la densidad por tipo de vegetación para el pecarí fue mayor en vegetación secundaria.

Posiblemente lo observado actualmente, es el efecto de la restricción de la cacería en REE desde 1990 cuando el área fue adquirida y convertida en reserva, lo que ha contribuido a un aumento en la densidad de los ungulados. Así mismo, la REE es un área formada principalmente por acahuales (reflejo de las explotaciones forestales y cultivo del chicle desde el siglo antepasado, así como la perturbación continua por incendios y huracanes), lo que puede beneficiar al venado cola blanca porque en este tipo de vegetación puede encontrar especies vegetales para su alimentación ya que es un animal selectivo. Además en estos sitios al ser muy densos encuentra alta cobertura de protección. Por otro lado, la cantidad de carreteras, caminos o asentamientos humanos alrededor del área pueden influir en gran medida en la densidad de ungulados, como sucede en Calakmul (Weber 2005). En el caso de la REE, es un sitio donde las vías de comunicación están muy alejadas, por tanto la influencia de las carreteras y poblados es menor y tiene suficiente cobertura para protegerlos.

\section{Distribución de los ungulados en base al tipo de vegetación}

Se observó que el venado cola blanca se distribuye más en el acahual probablemente porque es la vegetación más abundante y disponible. Además, este tipo de vegetación le ofrece especies vegetales que consumen, como las hojas de Metopium brownei, las flores y hojas de Lysiloma latisiliqua y las hojas de Gymnopodium floribundum, y se ha visto que en Calakmul, aunque en baja proporción, son parte de la dieta del venado (Weber 2005). Estas especies se 
encuentran en abundancia en el acahual. Además, al ser la vegetación más densa (Schultz 2003), posiblemente le sirve para resguardarse del clima y depredadores.

El venado cola blanca también se distribuye frecuentemente en el tintal, y se observó que su presencia se da según la disponibilidad de este tipo de vegetación en la Reserva. Su distribución posiblemente también se deba a que los tintales se encuentran en un área cercana a la estación de la REE, y probablemente existe un efecto de protección. Además, en este tipo de vegetación crecen especies importantes para su dieta (Weber 2003), como Haematoxylon campechianum de la cual consume los frutos, también las hojas y retoños de Eugenia winzerlingii, las hojas de Gymnopodium floribundum, las hojas de Metropium brownei y los frutos de Manilkara zapota, las cuales son especies que abundan en esta vegetación (Schultz 2003). Al ser un hábitat con una estructura densa, posiblemente también les está ofreciendo cobertura contra sus depredadores (Reyna-Hurtado \& Tanner 2005), además, se observaron algunos venados descansando debajo de los árboles de tinte. Así mismo, el tintal está estacionalmente inundado entre cuatro y seis meses en el año, y gran parte del suelo está formado de grietas y huecos de roca caliza (micro cenotes; Gómez Pompa et al. 2003), donde el agua se concentra cuando hay lluvias, por lo que también puede ser una fuente de agua disponible. En el caso de la selva, el venado cola blanca también está presente según la disponibilidad del tipo de vegetación y puede estar alimentándose de especies que forman la parte más importante de su dieta en la península, como los frutos de Manilkara zapota, las flores y hojas de Lysiloma latisiliquum, las hojas de Metopium brownei y los frutos y hojas de Brosimum alicastrum (Weber 2005). Sin embargo, y a pesar que se observa la presencia de rastros, sólo se encontraron individuos en las partes cercanas a tintales y curiosamente en el área de selva más profunda y lejos de la estación no se tuvo ninguna observación de ungulados, y esto probablemente se deba a la presencia de depredadores que pueden ahuyentarlos, ya que en zonas más adentradas a la selva se encontraron rastros de puma y jaguar. Las sabanas, en cambio, están siendo evitadas por el venado cola blanca, ya que se observaron pocos individuos y la presencia de rastros fue mínima. La sabana tiene pocas especies arbóreas (Schultz 2003) que le puedan estar sirviendo, por un lado, de alimento al cola blanca, ya que es un hábitat en donde no crecen suficientes árboles. Por otro lado, probablemente por ser áreas abiertas los venados las evitan porque no les proporcionan la cobertura de protección contra sus depredadores, elemento que es clave en el hábitat en que esté presente (Bello et al. 2001). Aparentemente, son usadas solo de paso para los venados cola blanca pues se observaron varias veces venados caminando y corriendo de los acahuales hacia la selva y viceversa, pasando de una selva densa a otra, posiblemente para esconderse y alimentarse. Probablemente las zonas inundadas de las sabanas también les proveen de agua. 
En el caso de los venados temazates no se encontraron diferencias en la distribución por tipo de vegetación. Esto posiblemente se debió a que el número de individuos fue muy bajo para determinar alguna preferencia. Sin embargo, se observaron más individuos distribuidos hacia el acahual y los tintales. Esto también lo encontraron Ávila (2003) al Sur de Quintana Roo y Bello et al. (2004) en Tabasco, mencionando que el temazate rojo puede observarse en acahuales y en pequeños claros de selva. Sin embargo, solo pudieron identificarse dos individuos del temazate café y tres del temazate rojo, y los nueve individuos restantes solamente se identificaron como Mazama spp; por lo que no se pudo determinar que especie de temazate fue observada con mayor frecuencia. Posiblemente el temazate café sea el más abundante, ya que en Calakmul esta especie tiene hábitos generalistas, similares al cola blanca y puede ser visto en sitios más perturbados como ocurrió en este estudio. Además se ha reportado que el temazate rojo es claramente un especialista de la selva mediana en Calakmul (Weber 2005).

El pecarí de collar se distribuye mayormente en el acahual, ya que éste posiblemente le provee de una mayor diversidad de especies vegetales como los frutos de Bursera simaruba, frutos de palmas, raíces, pequeños reptiles, artrópodos, entre otros (Sowls 1984, 1997, Martínez et al. 1995). Así mismo, entre la vegetación existen pequeños depósitos de agua que se llenan cuando hay lluvias y en una ocasión, se observó a un grupo tomando agua. Además, el acahual al ser muy denso puede proporcionarle cobertura contra depredadores. En la selva fueron observados muy pocos pecaríes, pero si se observaron varias veces restos de pelos y también heces fecales de grandes felinos compuestas de pelos de pecaríes. Lo que indica que éstos son parte importante de la dieta de los felinos (Aranda 2002, Olivera 2002) y por tanto los pecaríes parecen evitar zonas como las sabanas y tintales donde el riesgo de depredación pudiera ser mayor para ellos (Brown et al. 1999).

$\mathrm{Al}$ parecer existen algunos factores dentro del paisaje que también pueden estar influyendo en la distribución de los ungulados como lo es el "efecto estación", el cual significa que la distribución de individuos alrededor o cerca de la Estación de la Reserva es frecuente, porque perciben mayor seguridad, esto también pudo observarse en la Estación Biológica de Chamela (Mandujano, “com. pers."). Este efecto puede estar influyendo en la distribución del cola blanca, ya que varios registros fueron obtenidos alrededor de la estación, y éstos fueron principalmente hembras con crías y algunos juveniles. Posiblemente este efecto sea debido a que la estación les está proporcionando protección contra depredadores como el puma y el jaguar, pues los felinos sí son intimidados por la presencia de la gente, y es poco probable que se encuentren cerca de la estación, ya que los rastros registrados de estos felinos muestran una distribución lejana a la misma. También la estación les provee del recurso agua (Mandujano \& Gallina 1995), pues existe una fuente de agua que permanentemente está llena, además de que algunas veces los venados aprovechan los desperdicios de 
frutas que hay en la basura para alimentarse. Se ha visto que el venado cola blanca se acostumbra a la gente y se vuelve menos huidizo ante la presencia del humano cuando no es molestado (Emmons \& Feer 1990), por lo que esto posiblemente está determinando su distribución. En el caso de los temazates fue poco frecuente su presencia cerca de la estación al igual que los pecaríes, estos generalmente son más asustadizos y evitan al humano (Sowls 1984, 1997).

\section{Limitaciones del estudio y recomendaciones para futuros estudios}

La aplicación de métodos de muestreo de mamíferos medianos en selvas tropicales es complicada, ya que la mayoría de estas especies viven donde el hábitat es inaccesible para el humano, lo que dificulta su observación (Mandujano \& Gallina 1993, 1995, Weber 2000, 2005, Reyna-Hurtado 2002). Por tanto, el diseño de muestreo de este estudio fue planeado con base a las posibilidades y a la factibilidad de trabajar en un sitio como la REE. Esto implica posibles sesgos en las estimaciones de densidad, sobre todo en el caso de los temazates, ya que el empleo de senderos hace menos factible su observación debido a que la vegetación está perturbada y existen evidencias de que los temazates (Ávila 2003, Bello 2004, Weber 2005) necesitan de zonas boscosas o vegetación densa, aunque al parecer pueden adaptarse a zonas perturbadas. En el estudio se observaron pocos temazates sobre los senderos, siendo más frecuentes a distancias de $10 \mathrm{~m}$ del sendero. Por lo tanto, las densidades posiblemente están subestimadas.

En la REE solo existen cuatro senderos accesibles, y éstos mismos fueron recorridos varias veces, es decir, se recorrió la misma cantidad de kilómetros con varias repeticiones. Esto disminuye el área estudiada, y también las posibilidades de obtener mayores registros, además en estos casos, la posibilidad de ver al mismo animal es alta. Por otro lado, los senderos atraviesan los tipos de vegetación irregularmente, por lo que no se tuvo la misma área de muestreo por tipo de vegetación.

Así mismo, en algunas ocasiones los transectos fueron caminados por una persona y en otras con un acompañante, lo que pudo haber ocasionado cierto sesgo en la observación. Sin embargo, tuvo que realizarse así por medidas de seguridad, pero no siempre se contó con una persona para acompañante.

El haber muestreado en una superficie pequeña condujo a que el número de observaciones de ungulados fuera también pequeño, sobre todo para los temazates, por lo que los resultados obtenidos deben de tomarse con precaución, aunque sugieren tendencias que habrá que monitorear en el futuro, siendo lo encontrado en este estudio, una aproximación de la situación de las poblaciones de ungulados en la REE.

El resultado del conocimiento del área y la experiencia adquirida hace posible recomendar lo siguiente para futuros estudios: Realizar algunos transectos al azar para poder comparar la densidad obtenida en los senderos con densidades obtenidas con muestreos al azar. Para esto, es recomendable que el investigador esté apoyado 
por una persona de manera regular, de preferencia que conozca muy bien el área, como lo han hecho Weber $(2000,2005)$ y Reyna-Hurtado (2002), con los cazadores de Calakmul, ya que estos son expertos en el rastreo de animales. En el caso de la REE, sería preferible una persona de origen maya del poblado que conozca la reserva, ya que estas personas tienen un amplio conocimiento empírico de las selvas y pueden aportar valiosa información. También se recomienda caminar un mayor número de transectos pocas veces ya que de esta manera se abarcaría mayor área de muestreo y la probabilidad de ver los mismos animales se reduce. Así mismo, se deberían estratificar los transectos por tipo de vegetación para tener una comparación del área semejante.

Este trabajo, a pesar de sus limitaciones, proporciona una información ecológica básica útil para estudios futuros, ya que es el primero que se realiza con los ungulados en la REE. Además, este trabajo registró por primera vez a Mazama americana para esta región del norte de Quintana Roo. Sería de mucho interés entonces realizar estudios ecológicos más profundos de cada una de las especies de ungulados, así como de sus dietas y preferencias alimentarias y de la influencia de los ungulados en la regeneración de la selva de la REE. También se recomiendan estudios sobre depredación de ungulados y de su importancia como indicadores de la presencia de felinos en la REE.

\section{Implicaciones de manejo}

En diversos foros se ha discutido acerca de la importancia de las grandes áreas protegidas para la conservación. Sin embargo se sabe poco de la importancia de las áreas pequeñas como estrategia de conservar la vida silvestre (Gómez-Pompa \& Dirzo 1995). La REE es un área privada pequeña dedicada a la conservación. Esta se encuentra en proceso de regeneración. No existe presión antrópica como la cacería y posiblemente por ello es un área con mayor densidad de venados en comparación con otras áreas del trópico, lo cual es una consecuencia del efecto de protección que los ungulados perciben. Este "efecto reserva", permite que el área represente una fuente de recursos importante para otros animales (Meffe \& Carroll 1994), en este caso el venado cola blanca es la presa más importante en la dieta del puma, y el pecarí de collar la del jaguar (Aranda 2002) y posiblemente la presencia de felinos se deba a la alta densidad de presas. Por tanto, al elaborar estrategias para la conservación de ungulados se estará beneficiando también a sus depredadores (Sanderson et al. 2002). Así mismo, son herbívoros y frugívoros que pueden estar cumpliendo un papel de dispersores de semillas (Dirzo \& Miranda 1991), principalmente en partes más perturbadas como acahuales, influyendo de esta manera a la restauración de la vegetación de la REE. También, los ungulados de la reserva representan un reservorio de información genética que apoyará a la conservación de especies en el futuro 
(Meffe \& Carroll 1994). Tomando en cuenta lo anterior, la REE está teniendo una función importante en la conservación de los ungulados, que a pesar de la perturbación continua por incendios y huracanes, en la reserva se tiene una densidad relativamente alta, principalmente del venado cola blanca. Por lo tanto, la creación y conservación de pequeñas áreas como la REE, podría ser una alternativa más de conservación de la vida silvestre.

\section{AGRADECIMIENTOS}

Agradecemos al Consejo Nacional de Ciencia y Tecnología (CONACyT) y al Instituto de Ecología A. C, por el apoyo económico otorgado para la elaboración de esta investigación. Un especial agradecimiento a la Dra. Silvia Hernández Betancourt de la Universidad Autónoma de Yucatán y al Dr. Joaquín Bello de la Universidad Juárez Autónoma de Tabasco, por sus revisiones y comentarios que enriquecieron este estudio. A los compañeros biólogos Alberto González Gallina, Virginia Ramírez Sosa y Shona Goulding por su apoyo en el trabajo de campo. Al equipo de la REE, en especial a su director Dr. José María Ramos, al encargado de campo biólogo Juan Castillo, y a Esther, encargada de la logística, por las facilidades otorgadas en la Reserva.

\section{LITERATURA CITADA}

Allen, M. \& E. Rincón. 2003. The Changing global environment and the lowland Maya: past patterns and current dynamics. Pp. 13-29. In: A. Gomez-Pompa, M.F. Allen, S. Fedick \& J.J. Jimenez-Osornio, (Eds). The Lowland Maya Area. Three Millennia at the HumanWildland Interface. Food Productos Press, Nueva York.

Aranda, M. 2002. Importancia de los pecaríes para la conservación del jaguar en México Pp. 101-106. In: R.A. Medellín, C. Equihua, C.L.B. Chetkiewicz, P.G. Crawshaw Jr., A. Rabinowitz, K.H. Redford, J.G. Robinson, E.W. Sanderson \& A.B. Taber (Eds.) El Jaguar en el Nuevo Milenio. FCE; UNAM:WCS. México.

Ávila, G. 2003. Manejo de Fauna Silvestre en Bosques Tropicales por Ejidos Forestales de Quintana Roo. Tesis de Maestría. Colegio de Postgraduados. Estado de México.

Bello, J., S. Gallina \& M. Equihua. 2001. Characterization and habitat preferences by white-tailed deer in Mexico. J. Range. Manage. 54:537-545.

Bello, G. J. 2004. Mamíferos del estado de Tabasco: diversidad y especies amenazadas. Kuxulkab 9:5-9.

Bello-Gutiérrez, J., C. Guzmán-Aguirre \& J. Santos-Zúñiga. 2004. Aspectos ecológicos del venado cola blanca en la región sierra del estado de Tabasco. IX simposio de venados en México. FMVZ- UNAM. ANGADI. UAEH. Pachuca, Hidalgo.

Bisbal, F.J.E. 1994. Biología poblacional del venado matacán (Mazama spp.) (Artiodactyla: Cervidae) en Venezuela. Rev. Biol. Trop. 42:305-313.

Branan W.V. \& R.L. Marchinton. 1987. Reproductive Ecology of White-tailed and Red Brocket Deer in Suriname. Pp. 972-976. In: Wemmer C. M. (Ed.). Biology and Management of the Cervidae. Smithsonian Institution Press, Washington D.C. 
Brown, J.S., J.W. Laundré \& M. Gurung. 1999. The ecology of fear: optimal foraging, game theory, and trophic interactions. J. Mamm. 80:385-399.

Byers, C.R., R.K. Steinhosrt \& P.R. Krusman. 1984. Clarification of a technique for analysis of utilization-availability data. $J$. Wildl. Manage. 48:1050-1052.

Carrillo E., G. Wong \& A.D. Cuarón. 2000. Monitoring mammal populations in Costa Rican protected areas under different hunting restrictions. Conserv. Biol. 14: 1580- 1591.

De Landa, D. 1938. Relación de las Cosas de Yucatán. Ed. Dante. Mérida, Yucatán, México.

Dirzo, R. \& A. Miranda. 1991. Altered patterns of herbivory and diversity in the forest understory: A case study of the possible consequences of contemporary defaunation. Pp. 273-287. In: Peter W., M. Lewinsonn, G. Wilson and W. Woodruff (Eds.). Plant-animal interactions. Evolutionary ecology in tropical and temperate regions. John Wiley and Sons Inc.

Emmons, L.H. \& F. Feer. 1990. Neotropical rainforest mammals. A field guide. The University of Chicago Press. Chicago, IL.

Fragoso, J.M. 1997. Desapariciones locales del baquiro labiado (Tayassu pecari) en la Amazonía: migración, sobre-cosecha o epidemia?. Pp. 309-312. In: Fang, T.G., R. Bodmer, R. Aquino \& M. Valqui (Eds). Manejo de fauna silvestre en la Amazonía. UNAP, University of Florida UNDP/GEF e Instituto de Ecología. La Paz, Bolivia.

Gallina, S. 1990. El Venado Cola Blanca y su Habitat en la Michilia, Durango. Tesis de Doctorado. UNAM. Facultad de Ciencias. México, D. F.

Gómez-Pompa, A. 1998. La Conservación de la Biodiversidad de México: Mitos y Realidades. Boletín de la Sociedad Botánica de México 63:33-41.

Gómez-Pompa, A. 2000. El Edén Ecological Reserve Home Page. http://maya.ucr.edupril/el_eden/Home.html.

Gómez-Pompa, A. \& R. Dirzo. 1995. Las Reservas de la Biosfera y otras áreas naturales protegidas de México. SEMARNAP y CONABIO. México, D. F.

Gómez-Pompa, A., M.F. Allen, S.L. Fedick \& J.J. Jimenez-Osornio. 2003 The Lowland Maya Area. Three Millennia at the Human-Wildland Interface. Food Productos Press, Nueva York.

González-Marín, R.M. 2005. Los ungulados de la Reserva Ecológica El Edén, Quintana Roo, México: Un estudio sobre la densidad y distribución de Odocoileus virginianus yucatanensis, Mazama spp. y Pecari tajacu yucatanensis. Tesis de Maestría. Instituto de Ecología, A. C. Xalapa, Veracruz, México.

Hurtado-González, J.L. \& Bodmer, R.E. 2004. Assessing the sustainability of brocket deer hunting in the Tamshiyacu_Tahuayo Communal reserve, northern Peru. Biol. Conserv. 116:1-10.

Meffe, G.K. \& C.R. Carroll. 1994. Principles of Conservation Biology. Sinawer Associates, Inc. Publishes, Sunderland, Massachusetts.

Mandujano, S. 1999. Variation in herd size of collared peccaries in a Mexican tropical forest. The Southwest. Nat. 44:199-204.

Mandujano, S. \& S. Gallina. 1993. Densidad del venado cola blanca basada en conteos en transectos en un bosque tropical Jalisco. Acta Zool. Mex. 56: 1-37. 
Mandujano, S. \& S. Gallina. 1995. Comparison of deer censing methods in tropical dry forest. Wildl. Soc. Bull. 23:180-186.

Mandujano, S., S. Gallina, G. Arceo \& L.A. Pérez-Jiménez. 2004. Variación estacional del uso y preferencia de los tipos vegetacionales por el venado cola blanca en un bosque tropical de Jalisco. Acta Zool. Mex. (n. s.) 20: 45-67

Mandujano, S. \& V. Rico-Gray. 1991. Hunting, use, and knowledge of the biology of the white-tailed deer (Odocoileus virginianus Hays) by the Maya of Central Yucatán, México. J. of Ethnobiology 11:175-183.

Martínez-Romero, L.E. \& S. Mandujano. 1995. Hábitos alimentarios del pecarí de collar (Pecari tajacu) en un bosque tropical caducifolio de Jalisco, México. Acta Zool. Mex. (n. s.) 64: 1-20.

Merediz, G. 1995. Abundancia, distribución y posibilidades de aprovechamiento sustentable del jabalí de collar (Tayassu tajacu) y otras especies faunisticas de la zona Maya de Quintana Roo. Tesis de Licenciatura. Facultad de Ciencias. UNAM. México, D. F.

Naranjo, E.J. 2002. Population ecology and conservation of ungulates in the Lacandon forest, México. Ph. D. Dissertation. The University of Florida, Gainesville, Fl, USA. 146 p.

Naranjo, E., M. Guerra, R. Bodmer \& J. Bolaños. 2004. Subsistence hunting by three ethnic groups of the Lacandon Forest, Mexico. J. of Ethnobiology 24:233-253.

Olivera, T. 2002. Ecología comparativa de la alimentación del jaguar y del puma en el Neotrópico. Pp. 265-288. In: R.A. Medellín, C. Equihua, C.L.B. Chetkiewicz, P.G. Crawshaw Jr., A. Rabinowitz, K.H. Redford, J.G. Robinson, E.W. Sanderson \& A.B. Taber (Eds.) El Jaguar en el Nuevo Milenio. FCE; UNAM:WCS. México.

Pacheco, G. 2004. Densidad y distribución del pecari de collar (Pecari tajacu, Linnaeus 1758) en el Municipio de Tzucacab, Yucatán, México. VII Congreso Nacional de Mastozoología. San Cristóbal de las Casas, Chiapas. AMMAC: 64.

Pacheco-Figueroa, C.J. 2003. Situación actual del venado cola blanca (Odocoileus virginianus) y caracterización socioeconómica de los vecinos del Parque Nacional Barra Honda. Costa Rica. Tesis de Maestría. Universidad Nacional, Heredia, Costa Rica.

Reyna-Hurtado, R. 2002. Hunting effects on ungulate populations in Calakmul, Mexico. M.Sc. dissertation. University of Florida, Gainsville, Florida, USA.

Reyna-Hurtado, R. \& G.W. Tanner. 2005. Habitat Preferences of Ungulates in Hunted and Nonhunted Areas in the Calakmul Forest, Campeche, Mexico. Biotropica 34: 676-685.

Reyna-Hurtado, R. \& G.W. Tanner. 2007. Ungulate relative abundance in hunted and nonhunted sites in Calakmul Forest (Southern Mexico). Biodiv. Conserv. 16:743-757.

Robinette, W.L., C.M. Loveless \& D.A. Jones. 1974. Field tests of strip census methods. $J$. Wildl. Manage. 38: 81-96.

SAS Institute Inc. 2000. JMP IN Statistical Discovery Software, Versión 4. SAS Campus Drive. Cary, NC.

Schultz, G. 2003. Structure and Diversity of the Forests at the El Edén Ecological Reserve. Pp. 91-114. In: A. Gomez-Pompa, M.F. Allen, S. Fedick, \& J.J. Jimenez-Osornio, (Eds.). The Lowland Maya Area. Three Millennia at the Human-Wildland Interface Binghamton, NY: The Haworth Press.

Sowls, L.K. 1984. The Peccaries. The University of Arizona. Tucson, Arizona. 
Sowls, L.K. 1997. Javelinas and the other peccaries: their biology, management and use. 2nd. Ed. Texas A\&M University Press. College Station, Tx, USA.

Villarreal, J.G. 1990. Muestreo de poblaciones silvestres de venado cola blanca: método conteo físico nocturno con auxilio de luz artificial. Revista DUMAC 12:2-22.

Weber, M. 2005. Ecology and conservation of sympatric tropical deer populations in the Greater Calakmul Region, Mexico. School of Biological and Biomedical Sciences Ph.Sc. dissertation. University of Durham. Durham, United Kingdom.

Weber, M. \& S. González. 2003. Latin American deer diversity and conservation: A review of status and distribution. Ecoscience 10:443-454.

Wright, J.S., H. Zeballos., I. Domínguez., M.M. Gallardo, M.C. Moreno \& R. Ibáñez. 2000. Poachers alter mammal abundance, seed dispersal and seed predation in a neotropical forest. Conserv. Biol.14:227-239.

Zar, J. H. 1996. Biostatistical Analysis. Prentice Hall, New Jersey, USA.

Recibido: 12 de diciembre de 2006

Aceptado: 14 de noviembre de 2007 